\title{
Network Analysis of Cortical Visual Pathways Mapped with PET
}

\author{
A. R. McIntosh, ${ }^{1}$ C. L. Grady, ${ }^{1}$ L. G. Ungerleider, ${ }^{2}$ J. V. Haxby, ${ }^{1}$ S. I. Rapoport, ${ }^{1}$ and B. Horwitz ${ }^{1}$ \\ 'Laboratory of Neurosciences, National Institute on Aging, and 'Laboratory of Neuropsychology, National Institute of \\ Mental Health, National Institutes of Health, Bethesda, Maryland 20892
}

\begin{abstract}
Brain metabolic mapping techniques, such as positron emission tomography (PET), can provide information about the functional interactions within entire neural systems. With the large quantity of data that can accumulate from a mapping study, a network analysis, which makes sense of the complex interactions among neural elements, is necessary. A network analysis was performed on data obtained from a PET study that examined both the changes in regional cerebral blood flow (rCBF) and interregional correlations among human cortical areas during performance of an object vision (face matching) and spatial vision (dot-location matching) task. Brain areas for the network were selected based on regions showing significant $\mathrm{rCBF}$ or interregional correlations between tasks. Anterior temporal and frontal lobe regions were added to the network using a principal components analysis. Interactions among selected regions were quantified with structural equation modeling. In the structural equation models, connections between brain areas were based on known neuroanatomy and the interregional correlations were used to calculate path coefficients representing the magnitude of the influence of each directional path. The combination of the anatomical network and interregional correlations created a functional network for each task. The functional network for the right hemisphere showed that in the object vision task, dominant path influences were among occipitotemporal areas, while in the spatial vision task, occipitoparietal interactions were stronger. The network for the spatial vision task also had a strong feedback path from area 46 to occipital cortex, an effect that was absent in the object vision task. There were strong interactions between dorsal and ventral pathways in both networks. Functional networks for the left hemisphere did not differ between tasks. Networks for the interhemispheric interactions showed that the dominant pathway in the right hemisphere also had stronger effects on homologous left hemisphere areas and are consistent with a hypothesis that intrahemispheric interactions were greater in the right hemisphere in both tasks, and that these influences were transmitted callosally to the left hemisphere.
\end{abstract}

IKey words: structural equation modeling, human, spatial vision, object vision, cerebral blood flow, neural pathway, path analysis]

\footnotetext{
Received March 18, 1993; revised July 16, 1993; accepted July 21, 1993.

We thank Drs. C. DeCarli, N. J. Lobaugh, M. J. Mentis, and M. B. Schapiro for their critique of the early versions of the manuscript. A.R.M. was supported by the Natural Sciences and Engineering Research Council of Canada.

Correspondence should be addressed to Dr. A. R. McIntosh, Laboratory of Neurosciences, National Institutes on Aging, NIH Building, Room 6C414, Bethesda, MD 20892.

Copyright (c) 1994 Society for Neuroscience $0270-6474 / 94 / 140655-12 \$ 05.00 / 0$
}

Neuroscience research has been greatly aided by advances in functional neuroimaging techniques for both human subjects, using positron emission tomography (PET), and nonhuman subjects, using 2-deoxyglucose autoradiography (2-DG). These techniques make it possible to obtain functional maps of many regions simultaneously in a single brain, affording a singular advantage over traditional neuroscience methods. The analysis of mapping data is typically restricted to the comparison of average regional activity between experimental conditions, treating each brain region as an independent element, yet neural function likely relies upon the interactions between these elements (Luria, 1973; Damasio, 1989; Horwitz, 1989; Kosslyn and Intriligator, 1992). To make full use of mapping data, techniques that take into account the interactions between these elements are necessary.

Correlational analysis of metabolic mapping data has been used to examine the functional associations between different areas of the brain (Clark et al., 1984; Horwitz et al., 1984, 1992a; Soncrant et al., 1986; IIorwitz, 1989). This method assumes that brain areas functionally associated with one another during a particular condition will be strongly correlated, revealing functional interactions that are not obvious through simple examination of differences in mean regional activity. However, interpretations can become complicated when one wants to extend the discussion of the data beyond pairwise covariance relationships.

Recently, McIntosh and Gonzalez-Lima (1991, 1992a, 1993) demonstrated how structural equation modeling (also known as path analysis; Bollen, 1989; Jöreskog and Sörbom, 1989) could be used to quantify simultaneously the interactions among many brain areas. This computational method allows for the assessment of changes in the functional associations of entire systems. It has traditionally been used in genetics and social sciences for testing hypotheses about causal influences (Loehlin, 1987). When applied to neural systems, structural cquation modeling combines information about the anatomical pathways and the correlation coefficients of activity between brain regions to identify the functional pathways in a given experiment. The application of structural equation modeling to neural data assumes the pattern of correlations between brain areas is due to common influences and/or direct anatomical connections between them. The anatomical network model of the system is constructed from the known anatomical linkages between brain regions. The correlations between areas are decomposed to assign numerical weights (path coefficients) to the anatomical connections, which results in the functional network model. The strengths and signs of these path coefficients are compared across experimental conditions and used to identify task-specific functional interactions within the same anatomical network.

Structural equation modeling has been applied to 2-DG stud- 
ies of the rat auditory and visual system. The first application of structural equation modeling to $2-\mathrm{DG}$ data was from a study that examined auditory system activity during long-term habituation of the acoustic startle reflex (McIntosh and GonzalezLima, 1991). The auditory system functional network suggested that the interactions between parallel auditory pathways increase as a function of experience with the acoustic stimulus. The subsequent application was based on the examination of the rat visual system 2 -DG uptake in response to different stimulus conditions and arousal levels (McIntosh and GonzalezLima, 1992a). Functional network models of the visual system suggested that corticofugal influences change depending on stimulus conditions, and that arousal can modify the interactions between the geniculostriate and tectocortical visual subsystems. Structural cquation modcling has also been uscd to examine auditory system interactions in response to an acoustic stimulus trained as either a Pavlovian conditioned excitor or inhibitor (McIntosh and Gonzalez-Lima, 1993). The functional networks suggested that auditory system operations depend not only on the physical qualities of a stimulus, but also its acquired behavioral significance. In all applications, relationships between regions were demonstrated that were not obvious from the analysis of mean regional activity.

The present study extended the application of structural equation modeling to human neural systems mapped with PET using data from a regional cerebral blood flow (rCBF) study on object versus spatial vision (Haxby et al., 1991). It was noted that in humans, as in other primates (Ungerleider and Mishkin, 1982), object identification and spatial location are processed by two different cortical pathways. From the comparison of rCBF between tasks, ventral cortical areas were involved more with object identification and dorsal areas more with spatial location. These observations were furthered by analysis of interregional correlations that showed large correlations among posterior ventral regions in object vision and large correlations among posterior dorsal regions in spatial vision (Horwitz et al., 1992a). Furthermore, significant correlations among these posterior cortical areas were found only in the right hemisphere, while mean changes in rCBF were bilateral. It was suggested that the bilateral activation may have resulted from transcallosal influences of the right hemisphere on the left. This hypothesis received some confirmation through simulation network modeling (Horwitz et al., 1992b). From these results, the aims of the present study were, first, to construct a functional network to account for the right hemisphere correlations. Then, this model was compared to a similar circuit in the left hemisphere. Finally, an interhemispheric model was constructed to determine if the pattern of interactions was consistent with the hypothesis of a strong transcallosal influence from the right hemisphere in both tasks.

Part of this work has appeared in abstract form (McIntosh et al., 1993).

\section{Materials and Methods}

Network analysis, as we define it, is a technique that identifies neural systems that are the main functional group(s) in a given experiment and quantifies the interactions within these systems. First, the brain regions that define the system to be modeled are selected. This selection may be based on a system of interest (e.g., limbic system, auditory system) or by some preliminary covariance analysis where regions are grouped according to some common underlying dimensions (e.g., principal components analysis, factor analysis, discriminant analysis). For this analysis and the structural equation models, the covariances are computed between subjects within the same task (for further discussion of sources of variability in brain imaging studies, see Horwitz et al., 1992b). After the regions are selected, directional connections between the brain areas are determined based on neuroanatomy, and specify the anatomical network. The anatomical network and the interregional correlations are then used to quantify the interactions with structural equation modeling. This defines the functional network.

\section{PET data}

Data were obtained from a previous PET study designed to examine rCBF patterns related to object versus spatial visual functions, details of which have been presented elsewhere (Haxby et al., 1991; Horwitz et al., 1992a) but are summarized here. Seventeen young adult righthanded males (aged 19-34 years) performed three visual tasks during PET. Object vision was examined using a face-matching task, where the subject indicated which of two choice faces corresponded to the face in the simultaneously presented sample. The response was indicated by pressing a button with the thumb on the side corresponding to the correct choice. A dot-location matching task was used to examine spatial vision, where the sample stimulus consisted of a dot in a square of which one side was a double line. The choice stimuli were rotated $90^{\circ}$ or $180^{\circ}$ relative to the sample. The subject indicated which choice stimulus had the dot in the same location relative to the double line by pressing a button with the left or right thumb as in the face-matching task. The third task was a sensorimotor control task with three empty squares configured as in the other tasks. The subjects pressed the left and right buttons alternately in response to this stimulus. Six PET scans (Scanditronix PC1024-7B tomograph; Scanditronix, Uppsala, Sweden) were performed on each subject after a bolus injection of $30 \mathrm{mCi}$ of $\mathrm{H}_{2}{ }^{15} \mathrm{O}$ for each scan. Scanning sessions began and ended with the sensorimotor control task. Twelve of the 17 subjects performed each of the spatial vision and object vision tasks twice, and the order presentation of the tasks was counterbalanced. These tasks were performed only once by the other five subjects.

PET images were analyzed using a region-of-interest (ROI) template (Azari et al., 1992; Grady et al., 1992; Horwitz et al., 1992a) resulting in a total of 87 ROIs ( 42 bilateral, 3 midline) for each subject. Values assigned to the ROIs represented the average rCBF across the two scans (or the single-run value in the case of the five subjects who performed the tasks once), transformed by dividing rCBF by the whole-brain CBF (Horwitz et al., 1992a).

\section{Selection of brain regions}

In the initial applications of structural equation modeling, brain regions were identified a priori based on neural systems of theoretical interest (i.e., auditory and visual; McIntosh and Gonzalez-Lima, 1991, 1992a, 1993). For the present study, brain regions were selected by objective criteria, including regions that showed significant changes in $\mathrm{rCBF}$ and in the patterns of interregional correlations between conditions (Horwitz et al., 1992a). To identify additional regions that would extend the cortical network, a principal components analysis (PCA) with varimax rotation was used (Harman, 1969; Stevens, 1992). The assumption behind this approach was that brain areas operating together as a network should be related along a common dimension and could be identified using PCA. The PCA was done independently for each hemisphere and within each task since the interregional correlation analysis suggested that the relationships among cortical areas were different between tasks and between hemispheres (Horwitz et al., 1992a).

In choosing regions for the model, the same brain areas were ultimately selected for both tasks. This allowed the functional interactions to be compared within the same anatomical network. Components that appeared to define a network were retained, which in practice usually includes only the first few components. Given the focus on the neocortical visual system, motor regions were excluded from the models.

\section{Structural equation models}

Theory. The algorithms used for structural equation modeling attempt to account for an observed pattern of correlations based on the causal structure of the system (Loehlin, 1987; Bollen, 1989; Jöreskog and Sörbom, 1989). For example, consider the simplified "neural system" presented as a path diagram in Figure $1 a$. Brain areas A, B, C and D have particular anatomical connections given by the arrows, which provide the causal order of the system. The direct paths indicate that area $A$ projects to $B$ and $C$, area $B$ to $C$, and areas $B$ and $C$ project to $D$. Using 
path-tracing rules outlined by Wright for path analysis (as cited in Loehlin, 1987), the system can be represented by a set of path equations where the correlations between A, B, C, and D (Fig. 1b) are expressed as the sum of the compound paths connecting the four regions as shown in Figure $1 c$ (residual influences have been omitted for simplicity). The values for $\mathrm{v}, \mathrm{w}, \mathrm{x}, \mathrm{y}$, and $\mathrm{z}$ can be obtained through algebraic substitution and represent the path coefficients for the influences between structures through the anatomical connections.

An equivalent representation of the causal order of the system is by a set of structural equations rather than path equations (Fig. 1d). These structural equations can be related to path equations through derivation by converting the correlations back to variances and covariances (for the mathematical proof, see Loehlin, 1987). Structural equations specify the components of the variance for each variable, while path equations specify the components of each correlation coefficient. For these equations, $\mathrm{A}, \mathrm{B}, \mathrm{C}$, and $\mathrm{D}$ represent known parameters and are the variances for each region. The unknown parameters to be solved are $v, w, x, y$, 7., and each value for $\psi$ (residual influences). The solutions for the structural equations are obtained in a similar fashion to multiple regression, but rather than solving for each equation independently, they are considered simultaneously using matrix operations.

Mathematically, the goal of structural equation modeling is to obtain a solution to a set of structural equations that minimizes the differences between the observed covariance relationships and those that are irnplied by the solution. The process begins by giving each unknown parameter a starting value. These values are then used to start a series of successive "guesses" (iterations) for the parameters, given the correlations between variables. Using the "guesses," structural equation modeling programs compute the implied covariances and compare the values with the original covariances. Adjustments in the parameters are made based on the deviation of the implied covariances from the original covariances (optimization). This data-fitting procedure is performed until the deviation lies within an acceptable range.

The path coefficients are direct effects representing the influence of one brain region on another, controlling for the impact of other regions (Hayduk, 1987). Besides the direct effects, total effects can be examined. Total effects are the algebraic sum of direct and indirect effects (Loehlin, 1987; Bollen, 1989). Indirect effects represent the impact of a region transmitted through indirect routes. In Figure 1, the total effects of A on $\mathrm{C}$ would be the sum of the direct effect $(\mathrm{x})$ plus the indirect effects transmitted through B (vw). Evaluation of structural equation models in terms of total, direct, and indirect effects is referred to as effects decomposition (Hayduk, 1987). It is informative because it shows the total influence of an area or pathway and whether this influence is modified at any level of the system (McIntosh and Gonzalez-Lima, 1991, 1997b)

Model construction. The directional paths that defined the connections between the regions of the network were based on known neuroanatomy. However, becausc brain regions typically arc very highly interconnected, a compromise was reached between anatomical accuracy and the ability to interpret the model (McIntosh and Gonzalez-Lima, 1991, 1992b). Models that include all possible connections may be accurate, but not interpretable, whereas an excessively simplified model is of little utility. The other consideration in building the model was mathematical: if all connections were included there would have been too many unknown parameters relative to known quantities (correlations), making it impossible to solve the structural equations (Hayduk, 1987; McIntosh and Gonzalez-Lima, 1992b).

The path coefficients for the influences through the anatomical connections were then computed using LISREL (version 7, Scientific Software Inc.). The estimates for the path coefficients were initially obtained using two-stage least squares, and then, using these estimates as starting valucs, itcrativcly modificd using a maximum likclihood fit function (Jöreskog and Sörbom, 1989). Minimization was done with steepest-decent iterations and fine-tuned by Davidon-Fletcher-Powell iterations (Davidon, 1959; Fletcher and Powell, 1963; Jöreskog, 1973). Though this algorithm results in a reliable solution, solutions using different starting values were compared to ensure that the final solution was not a function of a local minimum.

Structural equation modeling allows for influences not measured or not measurable to be incorporated in the model as residuals. In the present model the representation of residuals is with the variable PSI $(\Psi)$ in LISREL terminology. These residual influences are best thought of as including the combined influences of areas outside the model and the influence of a brain region upon itself (McIntosh and GonzalezLima, 1992b). For each region a PSI variable was used to represent the a

STRUCTURAL MODEL

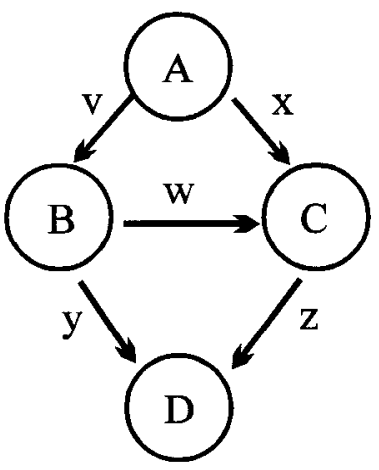

c

$$
\begin{aligned}
& \text { PATH Equations } \\
& r_{A B}=v \\
& r_{A C}=x+(v w) \\
& r_{A D}=(v y)+(x z)+(v w z) \\
& { }^{r_{B C}}=w+(v x) \\
& { }^{{ }_{B}}=y+(w z)+(v x z) \\
& { }^{r_{C D}}=z+(w y)+(x v y)
\end{aligned}
$$

Figure 1. Representation of the basic processes involved in structural equation modeling/path analysis. Panel $a$ is a path diagram of a "brain" with four areas $(A, B, C$, and $D)$, and the anatomical connections between them are indicated by arrows. Structural equation modeling/path analysis uses information about the anatomical connections (panel a) and the correlations of activity between regions (panel $b$ ) to determine the strength of influences through the connections. These influences are known as path coefficients $(v, w, x, y, z)$. Panel $c$ shows how the correlations between areas can be decomposed to solve for the path coefficients. The structural equations for the system presented in panel $d$ express the variance in activity in each area as a function of the weighted variance of other brain areas and some residual influence (indicated by $\psi$ ). Residuals were not represented in the path diagram or equations for simplicity. As a network becomes more complicated, solutions for the path coefficients are more easily obtained with least-squares or iterative methods using structural equations (see Materials and Methods).

influence of areas outside the model. In the present models, PSI was represented as a diagonal matrix so that residual influences were uncorrelated with each other.

Omnibus comparisons between tasks were done using the stacked model approach in LISREL (Jöreskog and Sörbom, 1989; McIntosh and Gonzalez-Lima, 1992a). Rather than estimating each model separately, the models were combined in a single program run. The process involved statistically comparing functional networks where path coefficients were constrained to be equal between conditions (null model) with those where the coefficients were allowed to differ (alternative model). Each model was assessed for its ability to reproduce the original correlation matrix through the $\chi^{2}$ goodness-of-fit statistic. The $\chi^{2}$ value is $(N-1)$ times the minimum value of the maximum-likelihood fit function, where $N$ is the total sample size (Jöreskog, 1973). A statistically insignificant $\chi^{2}$ goodness of fit indicates the model is able to reproduce the original correlation matrix reliably. If the $\chi^{2}$ value for the null model was significantly larger than the alternative model $\left(\chi_{\text {diff }}^{2}\right.$ test $)$, then the coefficients that were allowed to vary between conditions are statistically different. Generally, the $\chi^{2}{ }_{\text {diff }}$ test is used to determine if a modification to a model leads to a significant improvement in the fit of the model, and therefore the $\chi^{2}$ value should be statistically significant. In the case 
Table 1. Correlation matrix of normalized rCBF within each task

\begin{tabular}{|c|c|c|c|c|c|c|c|c|c|c|c|c|c|c|}
\hline & $17 / 18(r)$ & $19 v(r)$ & $37(r)$ & $21(\mathrm{r})$ & $19 d(r)$ & $7(\mathrm{r})$ & $46(r)$ & $17 / 18(1)$ & 1) $19 \mathrm{v}(1)$ & $37(1)$ & $21(1)$ & $19 \mathrm{~d}(1)$ & $7(1)$ & $46(1)$ \\
\hline \multicolumn{15}{|c|}{ Object vision task } \\
\hline $17 / 18(r)$ & 1.00 & & & & & & & & & & & & & \\
\hline $19 v(r)$ & 0.57 & 1.00 & & & & & & & & & & & & \\
\hline $21(r)$ & 0.12 & 0.49 & 0.34 & 1.00 & & & & & & & & & & \\
\hline $19 \mathrm{~d}(\mathrm{r})$ & 0.22 & 0.37 & 0.50 & 0.33 & 1.00 & & & & & & & & & \\
\hline $17 / 18(1)$ & 0.59 & 0.36 & 0.04 & 0.12 & -0.04 & 0.17 & -0.09 & 1.00 & & & & & & \\
\hline $19 v(1)$ & 0.67 & 0.32 & -0.12 & -0.13 & -0.38 & 0.33 & -0.23 & 0.55 & 1.00 & & & & & \\
\hline $37(1)$ & 0.03 & 0.30 & 0.56 & 0.22 & 0.27 & 0.17 & 0.00 & -0.03 & -0.16 & 1.00 & & & & \\
\hline $21(1)$ & -0.40 & 0.31 & 0.45 & 0.39 & 0.16 & 0.00 & -0.01 & -0.30 & -0.54 & 0.53 & 1.00 & & & \\
\hline $19 \mathrm{~d}(\mathrm{l})$ & 0.15 & -0.15 & -0.14 & 0.06 & 0.41 & 0.31 & -0.73 & -0.05 & 0.04 & -0.04 & -0.12 & 1.00 & & \\
\hline $19 v(r)$ & 0.27 & 1.00 & & & & & & & & & & & & \\
\hline $37(r)$ & 0.46 & 0.20 & 1.00 & & & & & & & & & & & \\
\hline $21(r)$ & 0.70 & 0.25 & 0.58 & 1.00 & & & & & & & & & & \\
\hline $19 \mathrm{~d}(\mathrm{r})$ & 0.31 & 0.40 & 0.42 & -0.01 & 1.00 & & & & & & & & & \\
\hline $7(\mathbf{r})$ & 0.16 & 0.43 & -0.18 & -0.33 & 0.60 & 1.00 & & & & & & & & \\
\hline $46(r)$ & -0.08 & 0.51 & -0.20 & -0.33 & 0.59 & 0.70 & 1.00 & & & & & & & \\
\hline $17 / 18(1)$ & 0.22 & 0.00 & 0.36 & 0.07 & 0.33 & -0.18 & -0.20 & 1.00 & & & & & & \\
\hline $19 v(l)$ & 0.40 & 0.09 & 0.40 & 0.47 & -0.03 & -0.02 & -0.40 & 0.20 & 1.00 & & & & & \\
\hline $37(1)$ & 0.60 & 0.18 & 0.59 & 0.82 & 0.00 & -0.33 & -0.51 & 0.01 & 0.48 & 1.00 & & & & \\
\hline $21(1)$ & 0.05 & 0.19 & 0.25 & 0.20 & -0.33 & -0.50 & -0.17 & -0.14 & -0.08 & 0.24 & 1.00 & & & \\
\hline
\end{tabular}

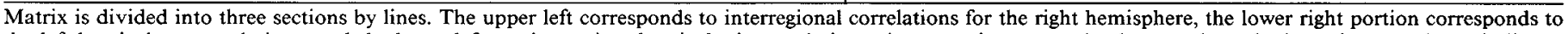

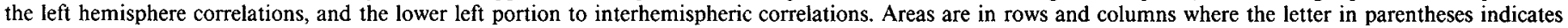
right or left hemisphere ( $\mathrm{r}$ or l). Designations of regions are given in terms of Brodmann areas.

of the present models, the modification was the removal of the betweengroup constraints on the path coefficient estimates.

The within-hemisphere functional networks for each task were constructed and compared. In the initial anatomical network it was decided that only the path coefficients for the feedforward connections from the occipital to the frontal lobe would be included. This minimized the number of unknown parameters to be estimated. A functional network accounting for the interhemispheric interactions was constructed in the final stage. For both intra- and interhemispheric functional networks, the influence of other connections (e.g., feedback connections) could be estimated if it significantly improved the fit of the model. The decision was based on modification indices of fixed coefficients (those that were not estimated in the initial run). Modification indices are computed from the partial derivatives of the fixed coefficients and give the expected improvement in the fit of the model if a cocfficient were freed (llayduk, 1987; Jöreskog and Sörbom, 1989). The final decision of whether to free the coefficient was based both on this statistical index and the theoretical feasibility.

Model interpretation. In a neural structural equation model, there is a temptation to interpret the sign of a path coefficient (positive or negative) as a reflection of excitatory or inhibitory influences in the electrophysiological sense, but this may not be accurate (McIntosh and Gonzalez-Lima, 1991). Instead, the path coefficients are interpreted in a manner similar to correlation or regression coefficients. A positive path coefficient means that a unit increase in the activity measure of one structure leads to a direct increase in the activity measure of structures it projects to, proportional to the size of the coefficient. Conversely, a negative path coefficient means that an increase in the activity measure in one structure leads to a direct, proportional decrease in the activity measure of structures it projects to. Positive and negative path coeffcients, therefore, measure the sign of the covariance relationships between components of the network.

Differences in path coefficients represent changes in the functional interaction between regions and can be of two types. One is represented as a difference in the sign of an effect without a marked difference in absolute magnitude. A difference in sign of the covariance relationship may be assumed to reflect a reversal in the interactions (from positive to negative or vice versa) within that pathway. This may be interpreted as a qualitative difference in the nature of the interaction between regions. On the other hand, if the difference is in absolute magnitude, but not sign, this is interpreted as a change in the strength of the influences conveyed through that pathway. This may suggest a quantitative shift (increase or decrease) in the influence of a pathway or structure on the activity of a system. This dichotomous classification aids in interpretation, but it is usually the case that changes in both sign and magnitude occur. While an individual path coefficient provides an indication of how a particular region is influencing another, the overall pattern of interrelations can give a clearer picture of how the system is interacting across conditions. Models can be interpreted based on well-known anatomical pathways (e.g., geniculostriate, septohippocampal) and whether there are discontinuities along these pathways indicated by sign changes or large changes in magnitude (McIntosh and Gonzalez-Lima, 1992b). Differences in either of these suggest an overall change in the relative influence of that pathway. 


\section{Object Vision}

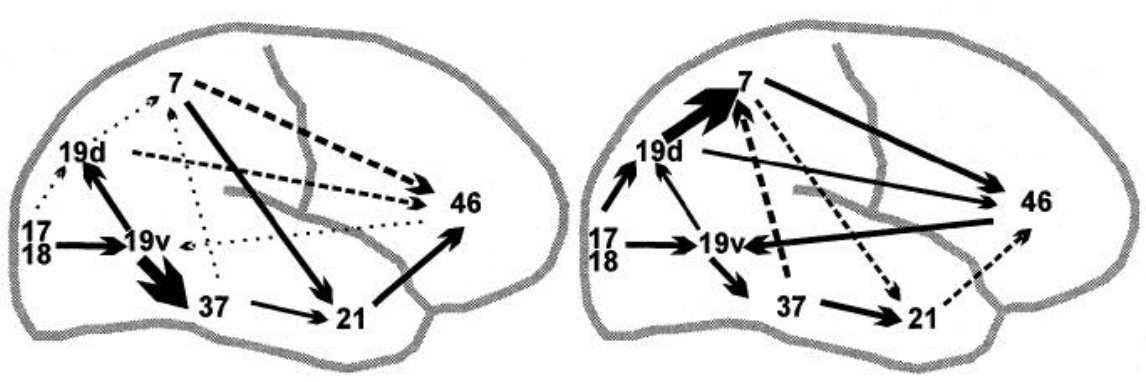

\section{Path Coefficients}

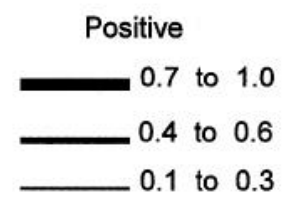

Figure 2. Graphic representation of the object vision (right) and spatial vision (left) functional networks in the right hemisphere. The magnitude of the direct effect is proportional to the arrow width for each path. Values for the width gradient are given in the legend at the bottom. Positive path coefficients are represented as solid black arrows, and negative as dashed arrows. Paths where the coefficient was at or near zero are depicted as a dotted line. The relative location of the brain region is somewhat distorted to maintain figure clarity.

\section{Results}

The regions that were ultimately selected, along with the interregional correlations, are presented in Table 1. Besides occipital, occipitotemporal, and parietal cortical areas, ROIs from the anterior temporal and frontal lobe were chosen from the PCA. Since part of the purpose in constructing the models was to account for the different pattern of significant correlations seen in the two hemispheres (Horwitz et al., 1992a), it was decided that the anatomical network constructed for the right hemisphere would be used for the left. The table presents both within- and between-hemisphere correlations. The selected ROIs in occipital and temporal cortices likely corresponded to Brodmann areas $17 / 18$, ventral and dorsal area $19(19 \mathrm{v}$ and $19 \mathrm{~d})$ in the occipital lobe, occipitotemporal area 37 , and anterior temporal area 21 . The parietal lobe region was designated as area 7 , and the frontal lobe region as area 46 . These designations were based on the approximated location of the template ROIs.

The connections between these areas were based on the neuroanatomy in nonhuman primates (Petrides and Pandya, 1988; Ungerleider et al., 1989; Pandya and Yeterian, 1990; Colby and Duhamel, 1991; Felleman and Van Essen, 1991; Knierim and Van Essen, 1992). To construct the anatomical network, we assumed homologies between areas $17 / 18$ and V1/V2, area 19 and V4, area 37 and TEO, and area 21 and TE. It needs to be emphasized that these anatomical designations should be regarded as tentative until they can be confirmed experimentally. Area $17 / 18$ formed the start of the network that extended into areas $19 \mathrm{v}$ and $19 \mathrm{~d}$. Areas 37 and 21 were regions along a ventral pathway, and area 7 along the dorsal pathway. Both the dorsal and ventral pathways continued into frontal cortex ending in area 46 . There were also connections between the dorsal and ventral pathways (area 37 to 7 , area 7 to 21 ) that were added based on modification indices from the null hypothesis model $\left[\chi_{\text {diff }}^{2}(1)=17.92, p<0.01\right.$ for area 37 to $7 ; \chi_{\text {diff }}^{2}(1)=24.80$, $p<0.01$ for area 7 to 21 ] and confirmed by anatomy (Pandya and Kuypers, 1969; Goldman-Rakic, 1988; Felleman and Van Essen, 1991). The PSI value for area $17 / 18$ was fixed at 1.0 since it had no paths to it in the model. PSIs for all other regions were fixed at 0.35 based on partial derivatives and modification indices. There was no indication that these residual influences were significantly different between tasks or hemispheres.

\section{Right hemisphere structural equation model}

Figure 2 graphically represents the functional networks for the right hemisphere in the two conditions. The values are the path coefficients obtained from a standardized solution where the size of a coefficient could range, in absolute value, from zero to one (Jöreskog and Sörbom, 1989). The omnibus test suggested that these models were distinct from each other $\left[\chi^{2}{ }_{\text {diff }}(11)=\right.$ $31.61, p<0.01]$.

As predicted by the $\mathrm{rCBF}$ and correlation analyses, interactions along the ventral pathway from area $19 \mathrm{v}$ extending into the frontal lobe were stronger in the object vision functional network, while interactions along the dorsal pathway from area $19 \mathrm{~d}$ to the frontal lobe were relatively stronger in the spatial vision functional network. Among posterior areas, the differences in path coefficients were mainly in magnitude. Occipitotemporal interactions between area $19 \mathrm{v}$ and area 37 were stronger in the object vision network while the impact of area $17 / 18$ to $19 \mathrm{~d}$ and the occipitoparietal influences from area $19 \mathrm{~d}$ to area 7 were stronger in the spatial vision network.

The anatomical model allowed for interactions between the dorsal and ventral pathways with connections from area 37 to area 7 and from area 7 to area 21. These interactions among the more anterior areas showed task-dependent differences in magnitude and sign. The temporoparietal influence of area 37 on area 7 was relatively stronger in the spatial vision network, and the parietotemporal influence of area 7 on area 21 showed a difference in sign between the two functional networks, with a positive influence in the object vision network and a negative sign in the spatial vision network. The influence of the dorsal and ventral pathways on frontal cortex was similar in magnitude for the two tasks, but the origin of the positive and negative influences differed, implying that the qualitative nature of interactions with the frontal lobe was different.

The other difference in the two functional networks was in the feedback path from area 46 to area $19 \mathrm{v}$. This path was not 


\begin{tabular}{|c|c|c|c|c|c|c|c|}
\hline & $17 / 18$ & $19 \mathrm{v}$ & 37 & 21 & $19 \mathrm{~d}$ & 7 & 46 \\
\hline \multicolumn{8}{|c|}{ Object vision } \\
\hline $17 / 18$ & $\overline{0}$ & $\overline{0}$ & $\overline{0}$ & $\overline{0}$ & $\overline{0}$ & $\overline{0}$ & $\overline{0}$ \\
\hline $19 \mathrm{v}$ & $\begin{array}{l}0.58 \\
0.58\end{array}$ & $\overline{0}$ & $\overline{0}$ & $\overline{0}$ & $\overline{0}$ & $\overline{0}$ & $\begin{array}{l}0.11 \\
0.10\end{array}$ \\
\hline 37 & $\overline{0.36}$ & $\begin{array}{l}0.62 \\
0.61\end{array}$ & $\overline{0}$ & $\overline{0}$ & $\overline{0}$ & $\overline{0}$ & $\overline{0}$ \\
\hline 21 & $\overline{0.12}$ & $\overline{0.21}$ & $\begin{array}{l}0.35 \\
0.32\end{array}$ & $\overline{0}$ & $\overline{0}$ & $\begin{array}{l}0.52 \\
0.53\end{array}$ & $\overline{0}$ \\
\hline $19 d$ & $\begin{array}{l}0 \\
0.22\end{array}$ & $\begin{array}{l}0.39 \\
0.39\end{array}$ & $\overline{0}$ & $\overline{0.02}$ & $\overline{0}$ & $\overline{0}$ & $\overline{0}$ \\
\hline 7 & $-\overline{0.01}$ & $-\overline{0.02}$ & $\begin{array}{l}-0.07 \\
-0.07\end{array}$ & $\overline{0}$ & $\begin{array}{l}0.07 \\
0\end{array}$ & $\overline{0}$ & $\overline{0}$ \\
\hline 46 & $-\overline{0.03}$ & $-\overline{0.05}$ & $-\overline{0.16}$ & $\begin{array}{l}0.44 \\
0.45\end{array}$ & $\begin{array}{l}-0.41 \\
-0.40\end{array}$ & $\begin{array}{c}-0.32 \\
0\end{array}$ & $\overline{0}$ \\
\hline \multicolumn{8}{|c|}{ Spatial vision } \\
\hline $17 / 18$ & $\overline{0}$ & $\overline{0}$ & $\overline{0}$ & $\overline{0}$ & $\overline{0}$ & $\overline{0}$ & $\overline{0}$ \\
\hline $19 v$ & $\begin{array}{l}0.32 \\
0.32\end{array}$ & $\overline{0}$ & $-\overline{0.24}$ & $-\overline{12}$ & $\overline{0.39}$ & $\overline{0.30}$ & $\begin{array}{l}0.56 \\
0.55\end{array}$ \\
\hline 37 & $\overline{0.10}$ & $\begin{array}{l}0.32 \\
0.31\end{array}$ & $\overline{0}$ & $\overline{0}$ & $\overline{0.12}$ & $\overline{0}$ & $\overline{0.18}$ \\
\hline 21 & $\overline{-} .05$ & $\overline{0.7}$ & $\begin{array}{l}0.55 \\
0.65\end{array}$ & $\overline{0}$ & $\begin{array}{c}-0.12 \\
-\end{array}$ & $\begin{array}{l}-0.23 \\
-0.17\end{array}$ & $\overline{0.11}$ \\
\hline $19 \mathrm{~d}$ & $\begin{array}{l}0.27 \\
0.32\end{array}$ & $\begin{array}{l}0.13 \\
0.22\end{array}$ & $\overline{0}$ & $\overline{0}$ & $\overline{0}$ & $\overline{0}$ & $\overline{0}$ \\
\hline 7 & $\overline{0.19}$ & $\overline{0}$ & $\begin{array}{r}-0.58 \\
0.56\end{array}$ & $\overline{0}$ & $\begin{array}{l}0.82 \\
0.79\end{array}$ & $\overline{0}$ & $\overline{0}$ \\
\hline 46 & $\overline{0.17}$ & $\overline{0}$ & $-\overline{43}$ & $\begin{array}{l}-0.22 \\
-0.21\end{array}$ & $\begin{array}{l}0.25 \\
0.69\end{array}$ & $\begin{array}{l}0.51 \\
0.54\end{array}$ & $\overline{0}$ \\
\hline
\end{tabular}

Within each row, upper value is the direct effect and lower value is the total effect. Direct effects were obtained by maximum-likelihood estimation, and total effects are the sum of direct and indirect influences. Rows list structures being affected and columns list the origin of the effect. Direct effects that were not estimated are indicated by a dash (-). Total effects less than 0.1 were set to zero. Region designation is as in Table 1 .

part of the initial anatomical network and was included based on a modification index in the spatial vision model. Its inclusion significantly decreased the overall $\chi^{2}$ goodness of fit for the model $\left[\chi_{\text {dir }}^{2}(2)=11.92, p<0.01\right]$. This path coefficient was strong in the spatial vision functional network but zero in object vision. We were unable to find evidence in the literature for a strong direct anatomical connection from area 46 to area 19 , although there are numerous indirect routes that could mediate this connection (e.g., thalamus or limbic cortices, Felleman and Van Essen, 1991; area 8, Nakamura et al., 1993). We therefore consider this to represent a functional influence mediated through structures not included in the present model. The between-task difference in this influence suggests that, regardless of the intervening structures, fecdback originating in the frontal lobe played a stronger role in spatial than in object vision.

Effects decomposition for both functional networks are presented in Table 2 . Two regions showed between-task differences in total effects. The spatial vision functional network showed larger total effects from areas 37, 19d, 7, and 46 on area $19 \mathrm{v}$. This was a result of the larger indirect effects from these areas through the feedback path from area 46 . Consistent with the weaker influence of dorsal paths in the object vision functional network, the total effect of area 7 on area $46(0.00)$ was smaller than its corresponding direct effect $(-0.32)$. The indirect effect of area 7 on 46 (through area 21 ) was positive $(0.23$; indirect $=$ total - direct), which reduced the negative direct effect. This did not occur in the spatial vision model.

\section{Left hemisphere structural equation model}

A graphic representation of the left hemisphere functional network is shown in Figure 3. The omnibus test suggested that path coefficients did not differ between tasks $\left[\chi_{\text {diff }}^{2}(11)=15.08, p>\right.$ $0.10]$, so the estimates of the coefficients were collapsed across the two conditions. The figure represents what could be thought of as the average interaction for the two conditions. Coefficients in both ventral and dorsal pathways from area $19 \mathrm{v}$ were positive until the final path to area 46 from areas 21 and 7 . The interaction between the two streams was also negative, and the coefficient for the feedback path from area 46 was zero. In some sense, this functional network seemed to be a blending of the features seen for each task in the right hemisphere network, but the magnitude of the effects was somewhat smaller.

\section{Interhemispheric structural equation model}

To construct a functional network accounting for the interactions between hemispheres, some simplifying restrictions were 


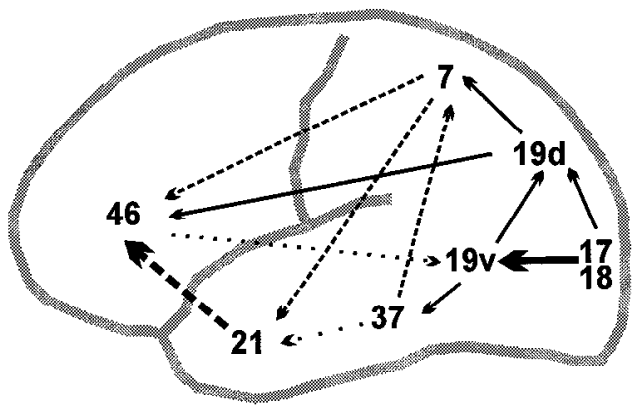

\section{Path Coefficients}
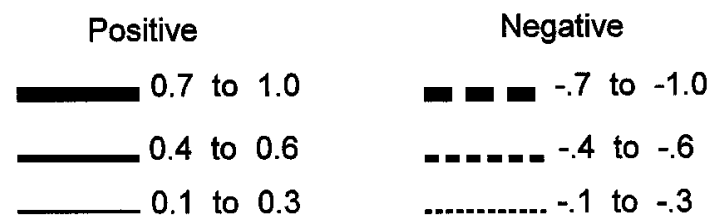

$\ldots \ldots$

Figure 3. Graphic representation of the left hemisphere functional network. The model did not differ between tasks, so the network represents the average of the two conditions. The magnitude of the direct effect is proportional to the arrow width for cach path. Valucs for the width gradient are as in Figure 2.

made to limit the number of coefficients estimated. First, the initial model was constructed with the interhemispheric interactions, represented as reciprocal influences, limited to homologous regions. The second restriction was to fix the coefficients for the intrahemispheric paths at the values from the previous analyses. These restrictions could be removed based on modification indices.

The final interhemispheric functional networks are presented in Figures 4 and 5. The omnibus comparison of the network suggested they were significantly different $\left[\chi_{\text {diff }}^{2}(17)=61.74, p\right.$ $<0.01]$. Additional paths from arca $19 \mathrm{~d}$ to arca 7 and area 46 to area 37 were included based on the modification indices for the spatial vision model. These inclusions significantly improved fit of the model [for area $19 \mathrm{~d}$ to area $7, \chi_{\text {diff }}^{2}(2)=13.86$, $p<0.01$; for area 46 to area $37, \chi_{\text {diff }}^{2}(2)=18.46, p<0.01$ ].

The dorsal and ventral pathways showed strong interactions between hemispheres. In the object vision lask (Fig. 4), the right hemisphere showed stronger positive influences from ventral regions on to left hemisphere areas, especially from areas 37 and 21 , whereas the left hemisphere showed weaker negative reciprocal influences. Dorsal regions showed weak interactions with a slight left hemisphere bias. The interhemispheric relation between dorsal and ventral pathways was reversed in the spatial vision functional network (Fig. 5). The dorsal areas showed greater interhemispheric interactions than ventral, with the right hemisphere having a larger influence on the left than the reverse. It was noteworthy that, unlike the more anterior areas, the interhemispheric interactions among area $17 / 18$ were equal in magnitude both between hemispheres and between tasks.

Effects decomposition revealed that differences between tasks in the total effects of left hemisphere regions were mainly in sign with the exception of area 46 (Table 3 ). Relative to the object vision functional network, the total effects in the spatial vision network of left area 46 on the contralateral hemisphere were higher for both ventral and dorsal cortical areas. For example, the total effect of left area 46 on right area 37 was -0.38 in the object vision network and -0.63 for the spatial vision network. For area 7 in the right hemisphere, the total effect of left area 46 was 0.00 for the object vision network and 0.37 for the spatial vision network. Since the direct effects of area 46 did not differ between tasks, the relatively greater total effects were a function of indirect influences mediated by the larger intrahemispheric feedback influence from area 46 in the right hemisphere for the spatial vision network. Overall, then, frontal lobe influence, from both left and right hemispheres, was greater in the spatial vision network. The lack of strong intrahemispheric frontal lobe feedback may have prevented such effects in the object vision task. Total effects of other regions were consistent with their dircet effects.

\section{Object Vision}

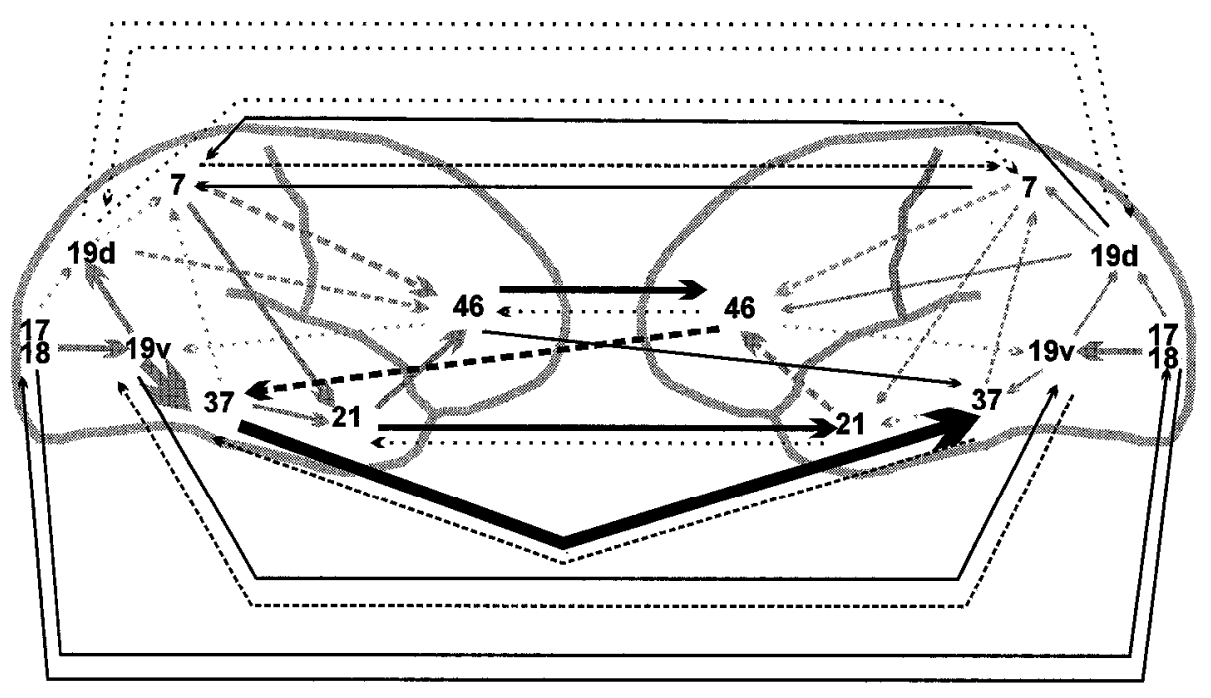

Figure 4. Graphic representation of the interhemispheric functional network for the object vision task. The within-hemisphere functional networks (Figs. 2,3) are depicted in the background in gray for comparison. The right hemisphere is on the left and left hemisphere on the right. The magnitude of the direct effect is proportional to the arrow width for each path. Values for the width gradient are as in Figure 2. 
Table 3. Effects decomposition of interrelations between hemispheres in the interhemisphere structural equation model

Right hemisphere on left

17/

$18(\mathrm{r}) \quad 19 \mathrm{v}(\mathrm{r}) \quad 37(\mathrm{r})$ $21(\mathrm{r}) \quad 19 \mathrm{~d}(\mathrm{r}) \quad 7(\mathrm{r})$
Left hemisphere on right

17

$\begin{array}{lllllll}18(\mathrm{l}) & 19 \mathrm{v}(\mathrm{l}) & 37(\mathrm{l}) & 21(\mathrm{l}) & 19 \mathrm{~d}(\mathrm{l}) & 7(\mathrm{l}) & 46(\mathrm{l})\end{array}$

\section{Object vision}

$17 / 18(\mathrm{r})$

$19 v(r)$

$37(r)$

$21(\mathbf{r})$

$19 \mathrm{~d}(\mathrm{r})$

$7(\mathrm{r})$

46(r)

\begin{tabular}{|c|c|c|c|c|c|c|c|}
\hline $17 / 18(1)$ & $\begin{array}{l}0.28 \\
0.31\end{array}$ & $\frac{-}{0}$ & $\frac{-}{0}$ & - & $\overline{0}$ & $\overline{0}$ & $\overline{0}$ \\
\hline $19 v(1)$ & $\overline{0.24}$ & $\begin{array}{l}0.29 \\
0.27\end{array}$ & $\overline{0}$ & $\overline{0}$ & $\frac{-}{0}$ & $\frac{-}{0}$ & $\overline{0}$ \\
\hline $37(1)$ & $\overline{0.28}$ & $\overline{0.42}$ & $\begin{array}{l}0.73 \\
0.52\end{array}$ & $\frac{-}{0}$ & $\frac{-}{0}$ & - & $\begin{array}{l}0.15 \\
0\end{array}$ \\
\hline $21(1)$ & $\overline{0}$ & $\frac{-}{0}$ & $\overline{0.19}$ & $\begin{array}{l}0.45 \\
0.46\end{array}$ & $\overline{0}$ & $\overline{0.26}$ & $\overline{0}$ \\
\hline $19 \mathrm{~d}(\mathrm{l})$ & $\overline{0.18}$ & $\overline{0.16}$ & $\frac{-}{0}$ & $\frac{-}{0}$ & $\begin{array}{l}0.29 \\
0.32\end{array}$ & - & $\frac{-}{0}$ \\
\hline $7(1)$ & $\frac{-}{0}$ & - & $\overline{0}$ & $\overline{0}$ & $\begin{array}{l}0.09 \\
0\end{array}$ & $\begin{array}{l}-0.15 \\
-0.16\end{array}$ & $\overline{0}$ \\
\hline $46(1)$ & $\frac{-}{0}$ & $\frac{-}{0}$ & $\frac{-}{0}$ & - & $-\overline{0.17}$ & $\frac{-}{0}$ & $\begin{array}{l}0.34 \\
0.34\end{array}$ \\
\hline
\end{tabular}

Spatial vision

$17 / 18(\mathrm{r})$

$19 v(r)$

$37(r)$

$21(\mathrm{r})$

$19 d(r)$

$7(r)$

$46(r)$

$\begin{array}{llllllll}17 / 18(1) & 0.11 & - & - & - & - & - & 0 \\ 0.11 & 0 & 0 & 0 & 0 & 0 & 0\end{array}$

$\begin{array}{llllllll}19 \mathrm{v}(\mathrm{l}) & - & 0.02 & - & - & - & - & -\end{array}$

$\begin{array}{llllllll}37(1) & - & - & 0.29 & - & - & - & -0.30\end{array}$

$\begin{array}{llllllll} & 0 & 0.13 & 0.41 & 0 & 0 & -0.10 & -0.19\end{array}$

$\begin{array}{llllllll}21(1) & - & - & - & 0.19 & - & - & -\end{array}$

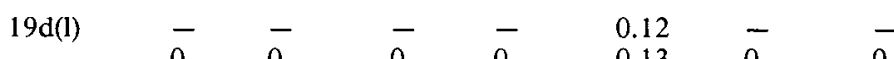

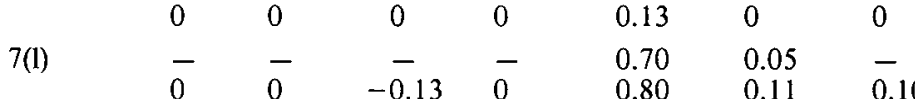

46(1) $\quad \begin{array}{lllllll} & - & - & - & - & -11 & 0.10\end{array}$

\begin{tabular}{|c|c|c|c|c|c|c|}
\hline 0.28 & - & - & - & - & - & - \\
\hline 0.31 & 0 & 0 & 0 & 0 & 0 & 0 \\
\hline- & -0.18 & - & - & - & - & - \\
\hline 0.10 & -0.17 & 0 & 0 & 0 & 0 & 0 \\
\hline- & - & -0.28 & - & - & - & -0.46 \\
\hline 0 & -0.15 & -0.25 & 0.16 & 0 & 0.14 & -0.38 \\
\hline- & - & - & 0.02 & - & - & - \\
\hline 0 & 0 & -0.11 & 0 & 0.22 & 0.25 & -0.11 \\
\hline- & - & - & - & 0.31 & - & - \\
\hline 0 & 0 & 0 & 0 & 0.34 & 0 & 0 \\
\hline- & - & - & - & 0.28 & 0.42 & - \\
\hline 0 & 0 & 0 & 0 & 0.41 & 0.38 & 0 \\
\hline- & - & - & - & - & - & -0.01 \\
\hline 0 & 0 & 0 & 0 & -0.17 & 0 & 0 \\
\hline
\end{tabular}




\section{Spatial Vision}

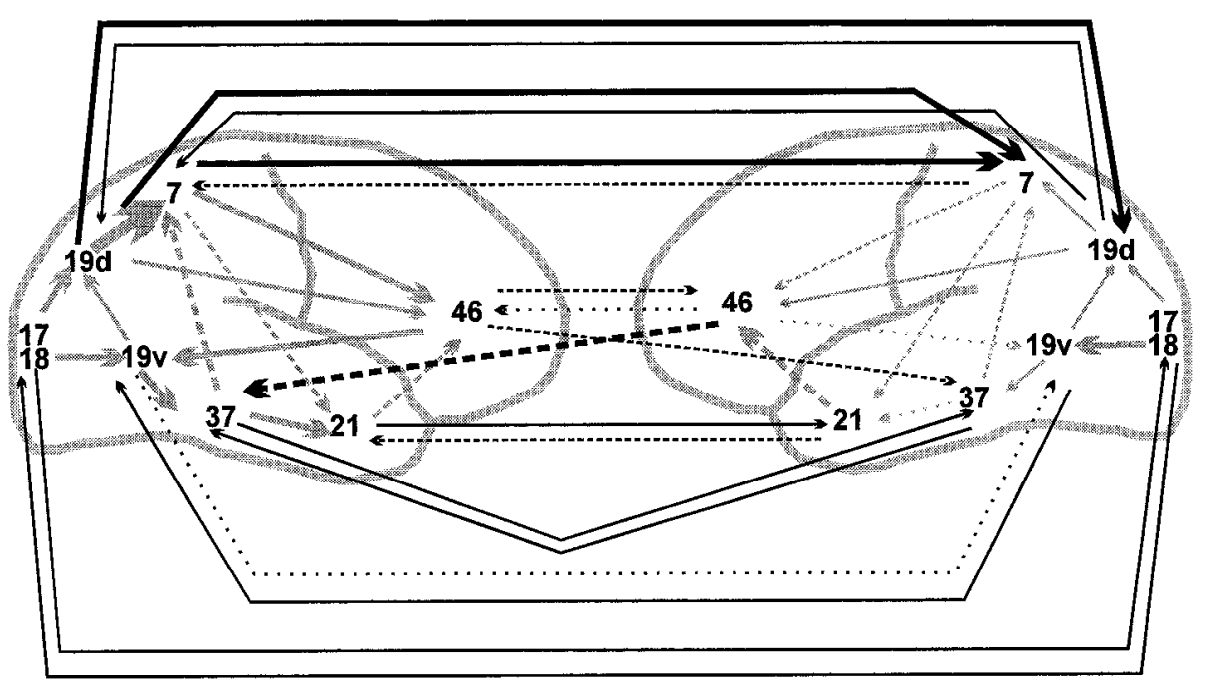

Figure 5. Graphic representation of the interhemispheric functional network for the spatial vision task. The within-hemisphere functional networks (Figs. 2,3) are depicted in the background in gray for comparison. The right hemisphere is on the left and left hemisphere on the right. The magnitude of the direct effect is proportional to the arrow width for each path. Values for the width gradient are as in Figure 2.

\section{Discussion}

The results of this study demonstrate how structural equation modeling can be used with PET data to provide information about the functional relationships among different brain areas. Structural equation modeling extended the correlational analysis by expressing these relationships in the context of directional influences in an anatomical network. The functional networks showed differences in the interactions of parallel cortical visual pathways in object and spatial vision and the interhemispheric interactions between these pathways. The networks included the influence of regions in the anterior temporal and frontal lobes that was not obvious by the traditional analysis of mean changes (i.e., these regions did not show a significant difference in rCBF in either task relative to the baseline control task).

Most of the differences between the two tasks involved the interactions within the right hemisphere. The right hemisphere functional network for object vision (face matching) showed strong continuous positive interactions along ventral pathways extending into frontal cortex. Dorsal interactions were discontinuous with zero path coefficients from area $17 / 18$ to 7 through $19 \mathrm{~d}$. These dorsal pathways were more strongly involved in the spatial vision (dot-location matching) task and were continuous into frontal cortex. Coefficients within the ventral pathway were positive and continuous up to the final link from area 21 to 46 , where the sign reversed to negative. The spatial vision functional network also had a strong feedback path coefficient from area 46 to $19 \mathrm{v}$. This path represented a functional influence possibly mediated through other regions that receive input from area 46 and project to area 19.

The right hemisphere functional networks are consistent with both the previous rCBF and correlational analyses, which showed that the ventral pathway was mainly involved in object identification and the dorsal pathway was stronger in spatial location (Haxby el al., 1991; Horwitz el al., 1992a). The network analysis quantified the directional interactions within and between path- ways in each task and demonstrated that the functional network extended beyond posterior cortical regions to include areas in the anterior temporal and frontal lobes. The involvement of the anterior temporal lobe in visual processing of objects has been noted in other PET rCBF studies using somewhat different stimuli (Sergent et al., 1992). The frontal lobe involvement was stronger in the spatial vision model, as illustrated by the strong feedback path. This could reflect the different requirements for the two match-to-sample tasks; the dot-location matching task may have required more processing steps, such as mental rotation and serial comparisons (Horwitz et al., 1992a).

Interactions between the dorsal and ventral pathways (from area 7 to 21 , and from arca 37 to 7 ) were present in the functional networks for both tasks, and showed differences in magnitude and sign between tasks. While the dorsal pathway was discontinuous in the object vision network, there was a strong positive direct effect from area 7 on the ventral pathway. This effect was strong, but negative, in the spatial vision network. Area 37 (in the ventral pathway) showed a strong influence on area 7 in the spatial vision network only. These interactions were not obvious in the correlation analysis.

The functional networks show that while the strongest positive interactions in each model may have preferentially been in one pathway, the parallel pathways were not functioning independently. The qualitative differences in the influence of "nondominant" pathways (e.g., area 21 to 46 in spatial vision and area 7 to 46 in object vision) and in the interactions between pathways suggest that, in addition to increases and decreases in activity, part of normal brain function entails modulation of the covariance relationship among different brain regions. Strong interactions between parallel pathways have been a consistent finding in all structural equation models of brain imaging data performed thus far (McIntosh and Gonzalez-Lima, 1991, 1992a, 1993) and emphasize that while a certain pathway or area may be critical for a particular function, operations in the intact brain involve interactions among many regions. Furthcrmore, neural networks based on the dorsal and ventral visual pathways have 
shown that behaviorally feasible models are obtained by including strong interactions among parallel, but functionally distinct, pathways (Otto et al., 1992; Tononi et al., 1992).

In both interhemispheric functional networks, the dominant right hemisphere pathway also showed a stronger influence contralaterally. The object vision network showed larger path coefficients among ventral areas from the right to the left hemisphere, while the spatial vision network had stronger coefficients among dorsal areas from the right to the left hemisphere. The correlational analysis of this data (Horwitz et al., 1992a) suggested that significant functional interactions occurred mainly in the right hemisphere, while the rCBF data suggested equal bilateral involvement. It was hypothesized that the reason for this discrepancy was that the right hemisphere was more involved in the tasks and the left hemisphere activations were due to the transcallosal influence of the right (Horwitz et al., 1992a). In support of this conjecture, dominant pathways in the right showed strong influences on the homologous left hemisphere areas in the structural equation models. This asymmetric relationship would not have been deduced from simple examination of the interhemispheric correlation matrices presented in Table 1 , but was quantified by the structural equation models. These results demonstrate how structural equation modeling can be used to confirm hypotheses about underlying network interactions.

\section{Evaluation of structural equation modeling application}

The purpose of the present analysis was to ascertain whether interactions among neocortical visual areas differed between the object and spatial vision task. Objective criteria (mean rCBF differences, correlations, and PCA) were used to select brain areas, but other networks also may have been involved in these tasks (e.g., attentional networks). As with any multivariate statistical analysis, however, the sample size limits the number of regions that can be simultaneously considered in an analysis. There are strategies for expanding structural equation models to include the interactions among smaller models (Hayduk, 1987). This was demonstrated in the present analysis for the interhemispheric model where intrahemispheric path coefficients were fixed at previously estimated values and the path coefficients for the interhemispheric interactions were freed. Such a strategy has also been employed in systems containing multiple reciprocal conncctions wherc path coefficients in one direction are estimated, then those estimates fixed and the estimates for the coefficients in the other direction determined (McIntosh and Gonzalez-Lima, 1992a). The reliability of estimates from this approach can be assessed by examination of the modification indices, which suggest whether the additions to base models change the original estimates of the path coefficients, or by doing the andysis in the reverse order and comparing the final solutions.

Models can be built hierarchically on a statistical basis using the $\chi_{\text {diff }}^{2}$ test. Given a choice between many potential connections, each could be evaluated for its statistical importance. The final model would contain those connections whose addition led to a significant improvement in the fit of the model. Constraints on this hierarchical approach could be imposed based on the neuroanatomical connections for the brain areas in the network and would give the minimal number of functional intcr- actions necessary to account for the interregional correlations. The maximum-likelihood estimates for the path coefficients are quite stable when new connections or structures are added to a model, but the stability of the final model will depend as much on the characteristics of the sample as it will on the algorithms for the structural equation models. The assumptions required for most inferential statistics need also be evaluated for structural equation modeling. Ill-conditioned data sets (e.g., those showing heteroscedasticity, outliers, and multicolinearity) will decrease the certainty of the maximum-likelihood estimates, making it more difficult to make inferential conclusions. Nonlinearities can pose difficulties in interpretation for the path coefficients if not detected, but nonlinear relationships can be incorporated into the models using approaches similar to a polynomial regression (Kenny and Judd, 1984).

Historically, structural equation modeling has been a tool for testing hypotheses about causal structure. In its application to neuroscience, the causal structure is established by the anatomy, but the models based on these data should still have a strong theoretical base. Models derived entirely from the data are vulnerable to sample-specific confounds and have been discouraged in applications of structural equation models to social science data (Freedman, 1987; MacCallum et al., 1992). Conversely, models based entirely on subjective decisions can lead to models that are not falsifiable. Analysis tools like structural equation modeling allow the researcher to combine objective and theoretical criteria in a meaningful way to generate or test hypotheses.

As PET has matured as a technicue for neuroscience research, data analysis techniques have also become more sophisticated. With the unique data set that is available from brain imaging, an analysis that looks at interregional interactions has become necessary to make full use of the information. Researchers have used different forms of covariance analysis, such as principal components or factor analysis (McLaughlin et al., 1992; Friston et al., 1993; Lagrèze et al., 1993), and multidimensional scaling (Goldenberg et al., 1989), to reveal the latent dimensions assumed to reflect the functional neural interactions (for some interesting extensions, see Moeller and Strother, 1991; Sun and Mazoyer, 1991). These techniques show each brain area's relation to the latent dimension. In this study, we used a PCA only as an initial step to help select brain regions that were functionally related to one another. Structural equation modeling allowed us to describe the nature of the functional relationships by expressing them as directional influences. Covariance relationships are decomposed within the constraints of an anatomical network, revealing asymmetric functional relationships (as in the interhemispheric models) that could not be extracted from the other aforementioned covariance analyses. This makes structural equation modeling distinctly different from other forms of data quantification: structural equation modeling, as used with brain imaging data, constructs systems-level neural models; it does not simply describe the relationships found with the brain imaging data set (Horwitz and McIntosh, 1993). Therefore, structural equation modeling is not an exploratory method; rather, it is a hypothesis-generating and hypothesistesting method. Finally, structural equation modeling should be useful in the examination of clinical populations, because one can now move from focusing on the pathology in single brain regions to evaluating how the cooperative interactions in brain networks are compromised by damage and discase. 


\section{References}

Azari NP, Rapoport SI, Salerno JA, Grady CL, Gonzalez-Aviles A, Schapiro MB, Horwitz B (1992) Interregional correlations of resting cerebral glucose metabolism in old and young women. Brain Res 589: 279-290.

Bollen KA (1989) Structural equations with latent variables. New York: Wiley.

Clark C, Kessler R, Buchsbaum M, Margolin R, Holcomb H (1984) Correlational methods for determining coupling of regional glucose metabolism: a pilot study. Biol Psychiatry 19:663-678.

Colby CL, Duhamel J-R (1991) Heterogeneity of extrastriate visual areas and multiple parietal areas in the macaque monkey. Neuropsychologia 29:517-537.

Damasio AR (1989) The brain binds entities and events by multiregional activation from convergence zones. Neural Comput 1:123132.

Davidon WC (1959) Variable metric method for minimization. A.E.C. Research and Development Report ANL5990. Argonne, IL: Argonne National Laboratory.

Felleman DJ, Van Esscn DC (1991) Distributcd hicrarchical proccssing in the primate cerebral cortex. Cereb Cortex 1:1-47.

Fletcher R, Powell MJD (1963) A rapidly convergent decent method for minimization. Comput $\mathrm{J}$ 6:163-168.

Freedman DA (1987) As others see us: a case study in path analysis. J Educ Stat 12:101-128.

Friston KJ, Fritch CD, Liddle PF, Frackowiak RSJ (1993) Functional connectivity: the principal-component analysis of large (PET) data sets. J Cereb Blood Flow Metab 13:5-14.

Goldenberg G, Poderka I, Steiner M, Willimes K, Suess E, Deecke L (1989) Regional cerebral blood flow pattern in visual imagery. Neuropsychologia 27:641-664.

Goldman-Rakic PS (1988) Topography of cognition: parallel distributed networks in primate association cortex. Annu Rev Neurosci 11: $137-156$.

Grady CL, Haxby JV, Horwitz B, Ungerleider LG, Schapiro MB, Carson RE, Herscovitch P, Mishkin M, Rapoport SI (1992) Dissociation of object and spatial vision in human extrastriate cortex: age-related changes in activation of regional cerebral blood flow measured with $\left[{ }^{15} \mathrm{O}\right]$ water and positron emission tomography. J Cognit Neurosci 4:23-34.

Harman HH (1969) Modern factor analysis. Chicago: University of Chicago.

Haxby JV, Grady CL, Horwitz B, Ungerleider LG, Mishkin M, Carson RE, Herscovitch P, Schapiro MB, Rapoport SI (1991) Dissociation of object and spatial visual processing pathways in human extrastriate cortex. Proc Natl Acad Sci USA 88:1621-1625.

Hayduk LA (1987) Structural equation modeling with t.ISRFi: essentials and advances. Baltimore, MD: John Hopkins UP.

Horwitz B (1989) Functional neural systems analyzed by use of interregional corrclations of glucose metabolism. In: Visuomotor coordination: amphibians, comparisons, models and robots (Ewert J-P, Arbib MA, eds), pp 873-892. New York: Plenum.

Horwitz B, McIntosh AR (1993) Network modeling and functional neuroimaging for mapping brain cognitive function. Ann Nucl Med 7:S90-S91.

Horwitz B, Duara R, Rapoport SI (1984) Intercorrelations of glucose metabolic rates between brain regions: application to healthy males in a state of reduced sensory input. J Cereb Blood Flow Metab 4:633678.

Horwitz B, Grady CL, Haxby JV, Schapiro MB, Rapoport SI, Ungerleider LG, Mishkin M (1992a) Functional associations among human posterior extrastriate brain regions during object and spatial vision. J Congnit Neurosci 4:311-322.

Horwitz B, Soncrant TT, Haxby JV (1992b) Covariance analysis of functional interactions in the brain using metabolic and blood flow data. In: Advances in metabolic mapping techniques for brain imaging of behavioral and learning functions (Gonzalez-Lima F, Finkenstädt T, Scheich H, eds), pp 189-218. NATO ASI Series, Dordrecht, Netherlands: Kluwer Academic.

Jöreskog KG (1973) A general method for estimating a linear structural equation system. In: Structural equation models in the social sciences (Goldberger AS, Duncanm OD, eds), pp 85-1 12. New York: Seminar.
Jöreskog KG, Sörbom D (1989) LISREL 7 user's reference guide. Mooresville, IN: Scientific Software.

Kenny DA, Judd CM (1984) Estimating nonlinear and interactive effects of latent variables. Psychol Bull 96:201-210.

Knierim JJ, Van Essen DC (1992) Visual cortex: cartography, connectivity, and concurrent processing. Curr Opin Neurobiol 2:150155.

Kosslyn SM, Intriligator JM (1992) Is cognitive neuropsychology plausible? The perils of sitting on a one-legged stool. J Cognit Neurosci 4:96-106.

Lagrèze HL, Hartmann A, Anzinger G, Schaub A, Deister A (1993) Functional cortical interactions patterns in visual perception and visuospatial problem solving. J Neurol Sci 114:25-35.

Loehlin JC (1987) Latent variable models: an introduction to factor, path and structural analysis. Hillsdale, $\mathrm{NJ}$ : Erlbaum.

Luria AR (1973) The working brain. New York: Basic.

MacCallum RC, Roznowski M, Necowitz LB (1992) Model modifications in covariance structure analysis: the problem of capitalization on chance. Psychol Bull 111:490-504.

McIntosh AR, Gonzalez-Lima F (1991) Structural modeling of functional ncural pathways mapped with 2-deoxyglucosc: cffects of acoustic startle habituation on the auditory system. Brain Res 547:295302.

McIntosh AR, Gonzalez-Lima F (1992a) Structural modeling of functional visual pathways mapped with 2-deoxyglucose: effects of patterned light and footshock. Brain Res 578:75-86.

McIntosh AR, Gonzalez-Lima (1992b) The application of structural modeling to metabolic mapping of functional neural systems. In: NATO ASI series: Advances in metabolic mapping techniques for brain imaging of behavioral and learning functions (Gonzalez-Lima F, Finkenstädt T, Scheich $H$, eds), pp 219-258. Dordrecht: Kluwer Academic.

McIntosh AR, Gonzalez-Lima F (1993) Network analysis of functional auditory pathways mapped with fluorodeoxyglucose: associative effects of a tone conditioned as a Pavlovian excitor or inhibitor. Brain Res, in press.

McIntosh AR, Grady CL, Ungerleider LG, Haxby JV, Horwitz B (1993) Network analysis of cortical visual pathways mapped with PET. J Cereb Blood Flow Metab 13:S523.

McLaughlin T, Steinberg B, Christensen B, Law I, Parving A, Friberg L (1992) Potential language and attentional networks revealed through factor analysis of rCBF data measured with SPECT. J Cereb Blood Flow Mctab 12:535-542.

Moeller JR, Strother SC (1991) A regional covariance approach to the analysis of functional patterns in positron emission tomographic data. J Cereb Blood Flow Metab 11:A121-A135.

Nakamura H, Gattass R, Desimone R, Ungerleider LG (1993) The modular organization of projections from areas $\mathrm{V} 1$ and $\mathrm{V} 2$ to areas $\mathrm{V} 4$ and TEO in macaques. $J$ Neurosci, in press.

Otto I, Grandguillaume P, Boutkhil L, Burnod Y, Guigon E (1992) Direct and indirect cooperation between temporal and parietal networks for invariant visual recognition. J Cognit Neurosci 4:35-57.

Pandya DN, Kuypers JM (1969) Cortico-cortical connections in the rhesus monkey. Brain Res 13:13-36.

Pandya DN, Yeterian EH (1990) Prefrontal cortex in relation to other cortical areas in rhesus monkey: architecture and connections. Prog Brain Res 85:63-94.

Petrides M, Pandya DN (1988) Association fibers to the frontal cortex from the superior temporal region in the rhesus monkey. I Comp Neurol 273:52-66.

Sergent J, Ohta S, MacDonald B (1992) Functional neuroanatomy of face and object processing. Brain 115:15-36.

Soncrant TT, Horwitz B, Holloway HW, Rapoport SI (1986) The pattern of functional coupling of brain regions in the awake rat. Brain Res 369:1-11.

Stevens J (1992) Applied multivariate statistics for the social sciences, 2d ed. Hillsdale, NJ: Erlbaum.

Sun H, Mazoyer BM (1991) Simulations of a Hopfield neural network based on cerebral glucose metabolism regional correlations measured by positron tomography. J Cereb Blood Flow Metab 11S2:S371.

Tononi G, Sporns O, Edelman GM (1992) Reentry and the problem of intergrating multiple cortical areas: simulation of dynamic integration in the visual system. Cereb Cortex 2:310-335. 
Ungerleider LG, Mishkin M (1982) Two cortical visual systems. In: Analysis of visual behavior (Ingle DJ, Goodale MA, Mansfield RJW, eds) pp 549-586. Cambridge, MA: MIT Press.
Ungerleider LG, Gaffan D, Pelak VS (1989) Projections from the inferior temporal cortex to prefrontal cortex via the uncinate fascicle in rhesus monkeys. Exp Brain Res 76:473-484. 